\title{
Representing externally positive systems through minimal eventually positive realizations
}

\author{
Claudio Altafini \\ Division of Automatic Control, Dept. of Electrical Engineering, \\ Linköping University, SE-58183, Sweden. \\ email: claudio.altafinieliu.se
}

\begin{abstract}
In order to investigate the cases in which an externally positive discrete-time system fails to have a minimal positive realization, in this paper we introduce the notion of minimal eventually positive realization, for which the state update matrix becomes positive after a certain power. This property captures the idea that in the impulse response of an externally positive system the state of a minimal realization may fail to be positive, but only transiently. It is shown in the paper that whenever a minimal eventually positive realization exists, then the sequence of Markov parameters of the impulse response admits decimated subsequences for which minimal positive realizations exist and can be obtained by downsampling the eventually positive realization.
\end{abstract}

Index terms - positive linear systems; minimal realization; eventually positive matrices; Perron-Frobenius theorem.

\section{INTRODUCTION}

It is well-known that an externally (i.e., input-output) positive linear system need not have an internally positive realization, and when it does have one this is typically nonminimal see [3], [5] for an introduction to the topic.

The scope of this paper is to suggest a class of minimal realizations which are not positive but eventually positive in the sense that the state update matrix is allowed to have some negative entries but it becomes positive after a certain power [1], [8]. These matrices still satisfy the Perron-Frobenius theorem, with both left and right dominant eigenvector in $\mathbb{R}_{+}^{n}$. Therefore in discrete-time they naturally lead to free evolutions of the state variables that become nonnegative after a certain number of steps. If in addition the reachable cone associated to the realization is contained in $\mathbb{R}_{+}^{n}$, then the entire state vector must become positive after a transient. Under assumptions such as strict pole dominance of the spectral radius, we shown in the paper that a discrete-time minimal realization $\{A, b, c\}$ in which $A$ is eventually positive is guaranteed to be externally positive. As for the converse, we provide constructive procedures to obtain a minimal eventually positive realization from a given externally positive system. While we do not have an explicit proof that every externally positive system admits a minimal realization of this type, it is tempting to conjecture that indeed it is so, at least in the case of transfer functions with a simple strictly dominant pole.

A consequence of the existence of a minimal eventually positive realization is that the sequence of Markov parameters that compose the impulse response has decimated subsequences for which a minimal positive realization exists, and can be found downsampling the given eventually positive realization. The number of steps it takes for $A$ to become positive gives a lower bound on the sought decimation factor.

If the Nyquist-Shannon sampling theorem states that one has to sample a system at sufficiently high frequency not to loose information on its signals, for externally positive systems admitting a minimal eventually positive realization our results show that one can always obtain a minimal positive realization provided one accepts to sample at sufficiently low frequency. The rationale is that strict pole dominance implies that any excursion from the dominant mode is only transient, and will disappear in time. This concerns in particular all excursions violating positivity, induced by nonzero initial conditions in the state space. The downsampled system will be "slower" that the original system, but its state can be guaranteed not to violate positivity, all while maintaining the minimality of the state space.

The rest of this paper is organized as follows: Section II recalls the basic linear algebraic notions associated to eventually positive matrices, which are then used in Section III to introduce eventually positive realizations in discrete-time. Constructive procedures are also given in this Section, while the downsampling of eventually positive realizations is studied in Section IV.

\section{LINEAR ALGEBRA BACKGROUND}

For a matrix $A=\left(a_{i j}\right) \in \mathbb{R}^{n \times n}$, in this paper $A \geq 0$ means $a_{i j} \geq 0$ for any $i, j \in 1, \ldots, n$, and $A \neq 0$, while $A>0$ means $a_{i j}>0$ for all $i, j=1, \ldots, n$. The matrix $A$ is nonnegative (resp. positive) if $A \geq 0$ (resp. $A>0$ ). This notation is used also for vectors. The spectrum of $A$ is denoted $(A)=\left\{\lambda_{1}(A), \ldots, \lambda_{n}(A)\right\}$, where $\lambda_{i}(A), i=$ $1, \ldots, n$, are the eigenvalues of $A$. The spectral radius of $A$, $\rho(A)$, is the smallest real positive number such that $\rho(A) \geq$ $\left|\lambda_{i}(A)\right|, \forall i=1, \ldots, n$.

\section{A. Eventually positive matrices}

Definition 1: A matrix $A \in \mathbb{R}^{n \times n}$ has the strong PerronFrobenius property if $\rho(A)$ is a simple positive eigenvalue of $A$ s.t. $\rho(A)>|\lambda|$ for every $\lambda \in(A), \lambda \neq \rho(A)$, and $v$, the right eigenvector relative to $\rho(A)$, is positive.

Denote $\mathcal{P} \mathcal{F}_{n}$ the set of matrices in $\mathbb{R}^{n \times n}$ that possess the strong Perron-Frobenius property. These properties are 
associated to a class of matrices called eventually positive [6], [7], [8], class that is strictly bigger than that of positive matrices, in the sense that the matrices can contain negative entries.

Definition 2: A real square matrix $A$ is said to be eventually positive if $\exists \eta_{o} \in \mathbb{N}$ such that $A^{\eta}>0$ for all $\eta \geq \eta_{o}$. The smallest integer $\eta_{o}$ of Definition 2 is called the power index of $A$. Following [10], eventually positive matrices will be denoted $A \stackrel{\vee}{>} 0$. For eventually positive matrices a necessary and sufficient condition for the fulfillment of the strong Perron-Frobenius property is available.

Theorem 1: ([8], Theorem 2.2) For $A \in \mathbb{R}^{n \times n}$ the following are equivalent:

1) Both $A, A^{T} \in \mathcal{P F}_{n}$;

2) $A \stackrel{\vee}{>} 0$;

3) $A^{T} \stackrel{\vee}{>} 0$.

\section{B. Invariant cones and eventually positive matrices}

A set $\mathcal{K} \subset \mathbb{R}^{n}$ is called a convex cone if $\alpha_{1} x_{1}+\alpha_{2} x_{2} \in \mathcal{K}$ $\forall x_{1}, x_{2} \in \mathcal{K}, \alpha_{1}, \alpha_{2} \geq 0 . \mathcal{K}$ is called solid if the interior of $\mathcal{K}$, $\operatorname{int}(\mathcal{K})$, is nonempty and pointed if $\mathcal{K} \cap(-\mathcal{K})=\{0\}$. A proper cone is a closed, pointed, solid cone. A cone is polyhedral if it can be expressed as the nonnegative combination of a finite number of generating vectors $\omega_{1}, \ldots, \omega_{\nu} \in \mathbb{R}^{n}$ :

$$
\mathcal{K}=\operatorname{cone}(\Omega)=\left\{x=\Omega \alpha=\sum_{i=1}^{\nu} \alpha_{i} \omega_{i}, \alpha_{i} \geq 0\right\},
$$

where $\Omega=\left[\omega_{1} \ldots \omega_{\nu}\right] \in \mathbb{R}^{n \times \nu}, \alpha=\left[\alpha_{1} \ldots \alpha_{\nu}\right]^{T} \in \mathbb{R}_{+}^{\nu}$. It is well-known that alternatively to the "vertices description" (1) for $\mathcal{K}$ one can use the "face description"

$$
\mathcal{K}=\{x \text { s. t. } \Gamma x \geq 0\}, \quad \Gamma \in \mathbb{R}^{\nu \times n} .
$$

The pair $\{\Omega, \Gamma\}$ forms a "double description pair" for $\mathcal{K}$. Let $\mathcal{K}^{*}=\left\{y \in \mathbb{R}^{n}\right.$ s. t. $\left.y^{T} x \geq 0 \forall x \in \mathcal{K}\right\}$ be the dual cone of $\mathcal{K}$. In terms of the double description pair $\{\Omega, \Gamma\}$, we have:

$$
\mathcal{K}^{*}=\left\{y \text { s. t. } y=\Gamma^{T} \beta, \beta \geq 0\right\}=\left\{y \text { s. t. } \Omega^{T} y \geq 0\right\},
$$

i.e., $\left\{\Gamma^{T}, \Omega^{T}\right\}$ is a double description pair for $\mathcal{K}^{*}$.

Given $A \in \mathbb{R}^{n \times n}$, the cone $\mathcal{K}$ is said $A$-invariant if $A \mathcal{K} \subseteq$ $\mathcal{K}$. For an $A$-invariant cone $\mathcal{K}, A$ is said $\mathcal{K}$-positive if $A(\mathcal{K} \backslash$ $\{0\}) \subseteq \operatorname{int}(\mathcal{K})$, i.e., $A$ maps any nonzero element of $\mathcal{K}$ into $\operatorname{int}(\mathcal{K})$. Notice that if $A$ is $\mathcal{K}$-positive then $A$ is $\mathcal{K}$-irreducible, i.e., it does not leave any of the faces of $\mathcal{K}$ invariant (except for $\{0\}$ and $\mathcal{K}$ itself). Theorem 1.3.16 of [4] says that $A$ that leaves $\mathcal{K}$ invariant is $\mathcal{K}$-irreducible if and only if $A$ has exactly one (up to scalar multiples) eigenvector in $\mathcal{K}$, and this vector is $\operatorname{in} \operatorname{int}(\mathcal{K}) . A$ is $\mathcal{K}$-positive if and only if $A^{T}$ is $\mathcal{K}^{*}$-positive [4].

The Perron-Frobenius theorem for invariant polyhedral cones can be found e.g. in [4] (Theorem 1.3.26) or [11] (Theorem 3.3).

Theorem 2: Given $A \in \mathbb{R}^{n \times n}$, the following are equivalent: (i)

1) $\exists$ a proper $A$-invariant polyhedral cone $\mathcal{K} \in \mathbb{R}^{n}$ for which $A$ is $\mathcal{K}$-positive;
2) $\rho(A)$ is a simple positive eigenvalue in $(A)$, and for each $\lambda \in(A), \lambda \neq \rho(A),|\lambda|<\rho(A)$.

Furthermore, for the right eigenvector $v$ relative to $\rho(A)$ it holds $v \in \operatorname{int}(\mathcal{K})$.

The following theorem links eventually positive matrices with invariant cones. Denote $\mathcal{K}^{\eta}$ the $\eta$-th iterated cone of $\mathcal{K}$ : $\mathcal{K}^{\eta}=A^{\eta} \mathcal{K}$.

Theorem 3: ([1], Theorem 5) $A \stackrel{\vee}{>} 0$ if and only if $\exists$ a proper polyhedral $A$-invariant cone $\mathcal{K}$ for which $A$ is $\mathcal{K}$ positive, and $\mathcal{K}$ is such that $\mathcal{K}^{\eta} \subset \operatorname{int}\left(\mathbb{R}_{+}^{n}\right) \cup\{0\},\left(\mathcal{K}^{*}\right)^{\eta} \subset$ $\operatorname{int}\left(\mathbb{R}_{+}^{n}\right) \cup\{0\} \forall \eta \geq \eta_{o}$.

By construction, the cone $\mathcal{K}$ of Theorem 3 contains no other eigenvector of $A$ than $v$. If instead of $A \stackrel{\vee}{>} 0$ we have the "one-sided" condition $A \in \mathcal{P} \mathcal{F}_{n}$, then Theorem 3 can be replaced by the following corollary.

Corollary 1: ([1], Corollary 2) $A \in \mathcal{P F}_{n}$ if and only if $\exists$ a proper polyhedral $A$-invariant cone $\mathcal{K}$ for which $A$ is $\mathcal{K}$ positive, and $\mathcal{K}$ is such that $\mathcal{K}^{\eta} \subset \operatorname{int}\left(\mathbb{R}_{+}^{n}\right) \cup\{0\} \forall \eta \geq \eta_{o}$.

\section{Discrete-time EVentually positive REALIZATIONS}

The discrete-time SISO linear system

$$
\begin{aligned}
x(k+1) & =A x(k)+b u(k) \quad k=0,1, \ldots \\
y(k) & =c x(k)
\end{aligned}
$$

is said externally positive if for any nonnegative input sequence $\{u(k)\}_{k=0}^{\infty}$ the forced output (in correspondence of $x(0)=0$ ) is nonnegative. A well-known necessary and sufficient condition for external positivity is that the impulse response $\left\{u(k)=\delta_{k}\right\}_{k=0}^{\infty}$ is nonnegative. The system (2) is said (internally, hereafter omitted) positive if for any nonnegative input sequence $\{u(k)\}_{k=0}^{\infty}$ and nonnegative initial condition $x(0)$, the state $x(k)$ and the output $y(k)$ are always nonnegative. A necessary and sufficient condition for positivity of (2) is that $A \geq 0, b \geq 0$ and $c \geq 0$ [5].

Consider the strictly proper, rational transfer function of order $n$

$$
H(z)=\frac{P(z)}{Q(z)}=\frac{p_{1} z^{n-1}+p_{2} z^{n-2}+\ldots+p_{n-1} z+p_{n}}{z^{n}+q_{1} z^{n-1}+\ldots+q_{n-1} z+q_{n}}
$$

where we assume that $P(z)$ and $Q(z)$ are coprime polynomials. Expanding (3) in terms of the Markov parameters $\{h(i)\}_{i=1}^{\infty}$ we can write

$$
H(z)=\sum_{i=1}^{\infty} h(i) z^{-i}
$$

where the recursive expression for the $\{h(i)\}_{i=1}^{\infty}$ is

$$
h(i)=-\sum_{j=1}^{i-1} q_{i-j} h(j)+p_{i} .
$$

A pole of $H(z)$ is called a dominating pole if its modulus is maximum among all poles of $H(z)$. It is called strictly dominating if it is dominating and all other poles of $H(z)$ have strictly smaller modulus.

A triplet $\{A, b, c\}$ is a realization of the strictly proper transfer rational function function $H(z)$ if $H(z)=c(z I-$ 
$A)^{-1} b$. A realization is minimal if and only if $\{A, b\}$ is controllable and $\{A, c\}$ observable. In this case $n$ denotes both the dimension of $A$ and the order of $H(z)$. A realization is positive if $\{A, b, c\}$ is a positive system.

Throughout this paper we will make the following assumption.

Assumption 1: $H(z)$ has a simple, strictly dominating real pole, denoted $\rho$, i.e., $Q(z)=Q^{\prime}(z)(z-\rho)$.

Under this assumption, any minimal realization $\{A, b, c\}$ has to have $\rho$ as a simple, strictly dominating real eigenvalue, i.e., $\rho=\rho(A)>|\lambda| \forall \lambda \in \operatorname{sp}\{A\}, \lambda \neq \rho(A)$ [3].

The following Theorem provides a necessary and sufficient condition for existence of a positive realization of $H(z)$.

Theorem 4: ([2], Theorems 2.1, 2.2 and Remark in between) Let $H(z)$ be a rational transfer function with minimal realization $\{A, b, c\}$. Then $H(z)$ has a positive realization iff $\exists$ a proper polyhedral cone $\mathcal{K}$ such that

1) $A \mathcal{K} \subseteq \mathcal{K}$

2) $b \in \mathcal{K}$

3) $c \in \mathcal{K}^{*}$.

In particular, in the case of simple strictly dominant real pole, it is known that a positive realization of some dimension $\nu \geq$ $n$ always exists (Theorem 31 of [5]) hence from Theorem 4, a proper $A$-invariant polyhedral cone $\mathcal{K}$ for which $b \in \mathcal{K}$ and $c \in \mathcal{K}^{*}$ always exists. Once $\mathcal{K}=\operatorname{cone}(\Omega)$ has been found [9], then $\Omega \in \mathbb{R}^{n \times \nu}, \nu \geq n$, can be used to form a $\nu$-dimensional positive realization $\left\{A_{p}, b_{p}, v_{p}\right\}$, where

$$
A \Omega=\Omega A_{p}, b=\Omega b_{p}, c_{p}=c \Omega .
$$

If $\nu>n$, the realization $\left\{A_{p}, b_{p}, v_{p}\right\}$ is nonminimal.

In this paper we introduce a class of minimal realizations which can be used to fill the gap between external positivity and (internal) positivity, at least under Assumption 1. We call these realizations eventually positive.

Definition 3: A realization $\left\{A_{e}, b_{e}, c_{e}\right\}$ is said eventually positive if $A_{e} \stackrel{\vee}{>} 0$ and $\exists$ an $A_{e}$-invariant cone $\mathcal{K}_{e}$ for which $A_{e}$ is $\mathcal{K}_{e}$-positive and such that $b_{e} \in \mathcal{K}_{e}, c_{e} \in \mathcal{K}_{e}^{*}$.

\section{A. A constructive algorithm}

For $H(z)$ satisfying Assumption 1, in this section we illustrate a procedure to construct an eventually positive minimal realization of $H(z)$. From Definition 3 and Theorem 1, in order to test whether a minimal realization $\{A, b, c\}$ is eventually positive we need to check the conditions $v>0$, $w>0$, where $v$ and $w$ are the right and left eigenvectors of $A$ relative to $\rho$. Two more conditions that can be added are $b \geq 0$ and $c \geq 0$.

An often used minimal realization of $H(z)$ is the following observability canonical form:

$A_{o}=\left[\begin{array}{cccc}0 & 1 & \ldots & 0 \\ \vdots & & \ddots & \\ 0 & & 0 & 1 \\ -q_{n} & \ldots & -q_{2} & -q_{1}\end{array}\right], \quad b_{o}=\left[\begin{array}{c}h(1) \\ h(2) \\ \vdots \\ h(n)\end{array}\right], \quad c_{o}=\left[\begin{array}{c}1 \\ 0 \\ \vdots \\ 0\end{array}\right]^{T}$.

$\left\{A_{o}, b_{o}, c_{o}\right\}$ is sometimes called Markov observability canonical form because of the Markov parameters appearing in $b_{o}$. In this case the polyhedral cone (hereafter $\mathcal{K}_{o}$ ) is called the Markov cone [2], [5] and it is generated by

$$
\Omega_{o}=\left[\begin{array}{cccc}
h(1) & h(2) & \ldots & h(\nu) \\
\vdots & \vdots & & \vdots \\
h(n) & h(n+1) & \ldots & h(\nu+n-1)
\end{array}\right]
$$

For the Markov observability canonical form, $b_{o} \geq 0$ and $c_{o} \geq 0$ by construction. The right eigenvector of $A_{o}$ relative to $\rho$ is easily computed to be $v_{o}=\left[\begin{array}{c}1 \\ \rho \\ \vdots \\ \rho^{n-1}\end{array}\right]>0$, while the left eigenvector $w_{o}$ has no fixed sign pattern. The condition $v_{o}>0$ guarantees that $A_{o} \in \mathcal{P F}_{n}$ and, from Corollary 1 , that $\mathcal{K}_{o}^{\eta}=\operatorname{cone}\left(\Omega_{o}\right)$ belongs to $\operatorname{int}\left(\mathbb{R}_{+}^{n}\right)$ for sufficiently high $\eta \in \mathbb{N}$. It however does not guarantee that also $A_{o}^{T} \in \mathcal{P} \mathcal{F}_{n}$, or that $\left(\mathcal{K}_{o}^{\eta}\right)^{*} \subset \operatorname{int}\left(\mathbb{R}_{+}^{n}\right)$. For that, one may have to "tilt" a little the $\left\{A_{o}, b_{o}, c_{o}\right\}$ realization, rendering $w_{o}$ positive while not changing the sign of $v_{o}, b_{o}$, and $c_{o}$. This can be done through operations with elementary matrices as in the following algorithm.

Recall that for a generic $n$-dimensional realization $\{A, b, c\}$, if $T$ is an invertible matrix, the change of basis performed via $T$ yields:

$$
\begin{aligned}
A^{\prime} & =T^{-1} A T \\
b^{\prime} & =T^{-1} b \\
c^{\prime} & =c T \\
v^{\prime} & =T^{-1} v \\
w^{\prime} & =T^{T} w .
\end{aligned}
$$

\section{Algorithm 1.}

Input: $A=A_{o}, b=b_{o}, c=c_{o}, v=v_{o}, w=w_{o}$.

Step 0: To begin with, notice that possibly by multiplying by $-I_{n}$, we can always assume that $w$ has at least $n / 2$ (or $(n+1) / 2$ if $n$ is odd) nonnegative entries.

Step 1: Consider an index $i$ for which $w_{i} \leq 0$. Two cases are possible:

Case 1: $\exists$ index $j \neq i, j \neq 1$, for which $w_{j}>0$ and

$$
0 \leq-\frac{w_{i}}{w_{j}}<\min \left(\frac{b_{j}}{b_{i}}, \frac{v_{j}}{v_{i}}\right)
$$

where by construction $-\frac{w_{i}}{w_{j}} \geq 0, \frac{v_{j}}{v_{i}}>0$, and $\frac{b_{j}}{b_{i}}>0$ when $b_{i} \neq 0\left(\frac{b_{j}}{b_{i}}=+\infty\right.$ otherwise, also admissible). In this case, choosing

$$
T^{(\xi)}=\left[\begin{array}{ccccc}
1 & 0 & \ldots & & 0 \\
0 & 1 & & & \\
\vdots & & \ddots & t_{j i}^{(\xi)} & \\
& & & & \\
& & & \ddots & 0 \\
0 & & & 0 & 1
\end{array}\right]=I_{n}+\left[t_{j i}^{(\xi)}\right]
$$


where $t_{j i}^{(\xi)} \in\left(-\frac{w_{i}}{w_{j}}, \min \left(\frac{b_{j}}{b_{i}}, \frac{v_{j}}{v_{i}}\right)\right)>0$, one gets in (6):

$$
\begin{aligned}
w_{i}^{\prime} & =w_{i}+t_{j i}^{(\xi)} w_{j}>0 \\
w_{j}^{\prime} & =w_{j}>0 \\
v_{i}^{\prime} & =v_{i}>0 \\
v_{j}^{\prime} & =-t_{j i}^{(\xi)} v_{i}+v_{j}>0 \\
b_{i}^{\prime} & =b_{i}>0 \\
b_{j}^{\prime} & =-t_{j i}^{(\xi)} b_{i}+b_{j}>0 \\
c_{i}^{\prime} & =c_{i} \\
c_{j}^{\prime} & =c_{j}=0 .
\end{aligned}
$$

i.e., the sign of $w_{i}$ has become positive without altering any of the other signs.

Case 2: No index $j \neq i$ exists for which $w_{j}>0$ and

(7) holds. In this case, choosing any index $j \neq i$, $j \neq 1$ for which $w_{j}>0$, it is possible to increase the corresponding $v_{j}$ and $b_{j}$ by combining the $j$-th row with any of the rows (indexed by $k$ ) having $w_{k}>0$ and $c_{k}=0$ (at least $n / 2-1$ such rows exist by construction). In this case the elementary matrix that can be used is

$$
T^{(\xi)}=I_{n}+\left[t_{j k}^{(\xi)}\right]
$$

with $-\frac{w_{k}}{w_{j}}<t_{j k}^{(\xi)}<0$, which yields

$$
\begin{aligned}
w_{j}^{\prime} & =w_{j}>0 \\
w_{k}^{\prime} & =t_{j k}^{(\xi)} w_{j}+w_{k}<w_{k} \\
v_{j}^{\prime} & =v_{j}-t_{j k}^{(\xi)} v_{k}>v_{j}>0 \\
v_{k}^{\prime} & =v_{k}>0 \\
b_{j}^{\prime} & =b_{j}-t_{j k}^{(\xi)} b_{k}>b_{j}>0 \\
b_{k}^{\prime} & =b_{k}>0 \\
c_{j}^{\prime} & =c_{j}=0 \\
c_{k}^{\prime} & =c_{k}=0
\end{aligned}
$$

If $v_{j}^{\prime}$ and $b_{j}^{\prime}$ are now sufficiently big that (7) holds, then Case 1 applies. If not, then another index $k$ must be chosen and Case 2 iterated.

Step 2: Repeat until $w_{j}^{\prime}>0 \forall j=1, \ldots, n$, or until Step 1 becomes unfeasible.

Step 3: If $w^{\prime}>0$ the Algorithm terminated successfully. In this case, if $T^{\left(\xi_{1}\right)}, \ldots, T^{\left(\xi_{\psi}\right)}$ are the $\xi_{\psi}$ elementary matrices used in Step 1 above, then the change of basis $T=T^{\left(\xi_{1}\right)} \cdots T^{\left(\xi_{\psi}\right)}$ yields the sought realization $\left\{A_{e}, b_{e}, c_{e}\right\}$.

The following Proposition shows that indeed $\left\{A_{e}, b_{e}, c_{e}\right\}$ constructed in this way is a minimal eventually positive realization of $H(z)$.

Proposition 1: Consider a strictly proper rational transfer function $H(z)$ obeying Assumption 1. Assume Algorithm 1 terminates successfully and consider the corresponding realization $\left\{A_{e}, b_{e}, c_{e}\right\}$. Then $\exists$ a proper polyhedral $A_{e^{-}}$ invariant cone $\mathcal{K}_{e}$ such that:

1) $\mathcal{K}_{e}^{\eta} \subset \operatorname{int}\left(\mathbb{R}_{+}^{n}\right) \cup\{0\}$ for $\eta \geq \eta_{o}$;
2) $\left(\mathcal{K}_{e}^{*}\right)^{\eta} \subset \operatorname{int}\left(\mathbb{R}_{+}^{n}\right) \cup\{0\}$ for $\eta \geq \eta_{o}$;

3) $A_{e}$ is $\mathcal{K}_{e}$-positive;

4) $b_{e} \in \mathcal{K}_{e}$

5) $c_{e} \in \mathcal{K}_{e}^{*}$.

Consequently, $\left\{A_{e}, b_{e}, c_{e}\right\}$ is a minimal eventually positive realization of $H(z)$.

Proof: Since $A_{e} \stackrel{\vee}{>} 0$, the first 3 items follow from Theorem 3. Applying the change of basis $T$ to the double description pair $\left(\Omega_{o}, \Gamma_{o}\right)$ of the Markov cone: $\Omega_{e}=T^{-1} \Omega_{o}$ and $\Gamma_{e}=T^{T} \Gamma_{o}$. By construction, $b_{e}=T^{-1} b_{o}$ where $b_{o}=$ $\Omega \alpha$ for some $\alpha \in \mathbb{R}_{+}^{\nu}$. Hence $b_{e}=T^{-1} \Omega_{o} \alpha=\Omega_{e} \alpha$, i.e., $b_{e} \in \mathcal{K}_{e}$. Similarly, $c_{e}=c_{o} T$, where $c^{T}=\Gamma_{o} \beta$ for some $\beta \in$ $\mathbb{R}_{+}^{\nu}$. Hence $c_{e}^{T}=T^{T} \Gamma_{o} \beta=\Gamma_{e} \beta$ i.e., $c_{e} \in \mathcal{K}_{e}^{*}$. Therefore $\left\{A_{e}, b_{e}, c_{e}\right\}$ is an eventually positive realization of $H(z)$. Minimality of this realization follows from invariance of the minimality property to a change of basis.

It follows from Proposition 1 and from $A_{e^{-}}$ invariance of $\mathcal{K}_{e}$ that for the reachability cone $\mathcal{R}_{e}=\operatorname{cone}\left(b_{e}, A_{e} b_{e}, A_{e}^{2} b_{e}, \ldots\right)$ of the system $\left\{A_{e}, b_{e}, c_{e}\right\}$ it holds:

$$
\mathcal{R}_{e} \subset \mathcal{K}_{e}
$$

Recall that the solution of a linear discrete-time system can be split into free and forced evolution. For $\left\{A_{e}, b_{e}, c_{e}\right\}$ one gets:

$$
x(k)=x_{o}(k)+x_{f}(k)=A_{e}^{k} x(0)+\sum_{j=0}^{k-1} A_{e}^{k-j-1} b_{e} u(j) .
$$

Proposition 2: Consider a minimal eventually positive realization $\left\{A_{e}, b_{e}, c_{e}\right\}$ of $H(z)$. Then $\exists \eta_{o} \in \mathbb{N}$ such that $x_{o}(k) \geq 0 \forall k \geq \eta_{o}, \forall x(0) \in \mathbb{R}_{+}^{n}$. If in addition $\mathcal{R}_{e} \subset \mathbb{R}_{+}^{n}$, then $x(k) \geq 0 \forall k \geq \eta_{o}, \forall x(0) \in \mathbb{R}_{+}^{n}, \forall u(k) \in \mathbb{R}_{+}$.

Proof: $A_{e} \stackrel{\vee}{>} 0$ implies $A_{e}^{k}>0 \forall k \geq \eta_{o}$, hence $x_{o}(k)=$ $A_{e}^{k} x(0) \geq 0 \forall x(0) \in \mathbb{R}_{+}^{n}$. Since $b_{e}$ can have any sign, an analogous property does not hold in general for the forced evolution $x_{f}(k)$. However, $\mathcal{R}_{e} \subset \mathbb{R}_{+}^{n}$ implies $x_{f}(k) \geq 0 \forall k$.

The following theorem summarizes the main result so far.

Theorem 5: Consider a strictly proper rational transfer function $H(z)$ obeying Assumption 1. If $H(z)$ admits a minimal eventually positive realization then it is externally positive. Conversely, if $H(z)$ is externally positive and Algorithm 1 terminates successfully, then $H(z)$ has a minimal eventually positive realization.

Proof: Consider an eventually positive realization and the corresponding $A_{e}$-invariant polyhedral cone $\mathcal{K}_{e}$. From $b_{e} \in \mathcal{K}_{e}$ and $c_{e} \in \mathcal{K}_{e}^{*}$, it follows that $c_{e} b_{e} \geq 0 . A_{e^{-}}$ invariance then implies that $A_{e}^{k} b_{e} \in \mathcal{K}_{e} \forall k=1,2, \ldots$ and therefore that $c_{e} A_{e}^{k} b_{e} \geq 0$. Conversely, Proposition 1 implies that success in Algorithm 1 yields an eventually positive realization $\left\{A_{e}, b_{e}, c_{e}\right\}$. Minimality also follows from Proposition 1. 


\section{B. Examples}

Example 1: Consider the following transfer function

$$
H(z)=\frac{0.1048 z^{3}+0.1312 z^{2}-0.02171 z-0.01499}{z^{5}-0.963 z^{4}-0.0582 z^{3}+0.035 z^{2}-0.0105 z-0.0033}
$$

Asd $\rho=1$, the Markov parameters are all nonnegative and "stabilize" at $\lim _{k \rightarrow \infty} h(k)=0.193$, we can conclude that $H(z)$ is externally positive. The Markov observability canonical form $\left\{A_{O_{2}}, b_{O_{2}}, c_{O_{2}}\right\}$ has $v_{O_{2}}=1$ and

$$
w_{o_{2}}=\left[\begin{array}{lllll}
0.0033 & 0.0138 & -0.0212 & 0.0369 & 0.9990
\end{array}\right]^{T},
$$

hence the dual cone $\left(\mathcal{K}_{e}^{*}\right)^{\eta}$ is not contained in $\mathbb{R}_{+}^{5}$ for any $\eta$. However, transforming this canonical form as in (6) with $T=I_{5}+\left[t_{43}\right], t_{43}=0.6076$, one gets

$$
A_{e}=\left[\begin{array}{ccccc}
0 & 1 & 0 & 0 & 0 \\
0 & 0 & 1 & 0 & 0 \\
0 & 0 & 0.6076 & 1 & 0 \\
0 & 0 & -0.3692 & -0.6076 & 1 \\
0.0033 & 0.0105 & 0.0003 & 0.0582 & 0.9631
\end{array}\right]
$$

which has positive right and left dominant eigenvectors

$$
v_{e}=\left[\begin{array}{c}
1 \\
1 \\
1 \\
0.3924 \\
1
\end{array}\right], \quad w_{e}=\left[\begin{array}{c}
0.0033 \\
0.0138 \\
0.0012 \\
0.0369 \\
1
\end{array}\right]
$$

$A_{e}$ is eventually positive, with a power index $\eta_{o}=8$, hence the $\left\{A_{e}, b_{e}, c_{e}\right\}$ realization is eventually positive. For it, $c_{e}=c_{o}$ and

$$
b_{e}=\left[\begin{array}{c}
0 \\
0.1048 \\
0.2321 \\
0.0669 \\
0.1951
\end{array}\right], \quad \text { while } \quad b_{o}=\left[\begin{array}{c}
0 \\
0.1048 \\
0.2321 \\
0.2079 \\
0.1951
\end{array}\right]
$$

where $b_{o}$ is the input matrix in the original Markov observability canonical form. In this case, altering (lowering) one of the Markov parameters has been enough to "compensate" the negative entry in the left eigenvector of $A_{o}$. A simulation of the impulse response for this eventually positive realization is shown in Fig. 1. It shows the transient violation of positivity of the state vector, which is not "visible" at the output. Since $b_{e} \geq 0$ and $A_{e}^{k} b_{e} \geq 0 \forall k$, in this case strict dominance implies that the violation of positivity must necessarily be transient for any nonnegative $\{u(k)\}_{k=0}^{\infty}$.

Example 2: It is worth observing that the power index of an eventually positive realization may vary among different eventually positive realizations of the same externally positive transfer function. Consider again Example 1. If instead of using the change of basis $T$ we use the following $T_{2}=I_{5}+\left[t_{53}\right]$ with $t_{53}=0.1032$, then we get another eventually positive realization, with

$$
A_{e_{2}}=\left[\begin{array}{ccccc}
0 & 1 & 0 & 0 & 0 \\
0 & 0 & 1 & 0 & 0 \\
0 & 0 & 0 & 1 & 0 \\
0 & 0 & 0.1032 & 0 & 1 \\
0.0033 & 0.0105 & 0.0643 & -0.0450 & 0.9631
\end{array}\right],
$$

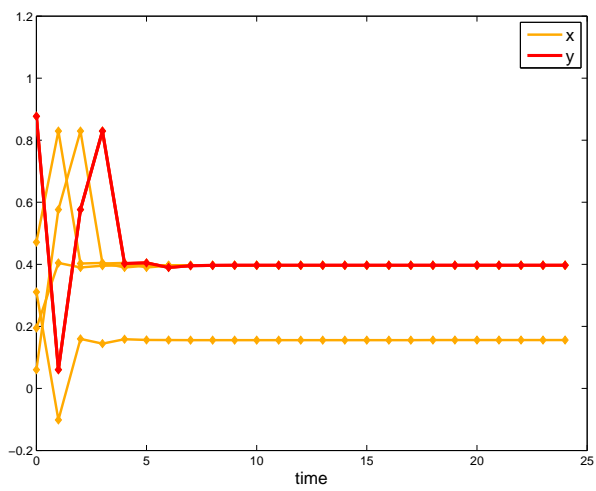

Fig. 1. Example 1. A typical dynamical behavior of a minimal eventually positive realization. When the initial conditions are nonzero but positive, the impulse response yields a positive output, but one of the state variables has a transient which does not remain positive.

of right and left dominant eigenvectors

$$
v_{e_{2}}=\left[\begin{array}{c}
1 \\
1 \\
1 \\
1 \\
0.8968
\end{array}\right], \quad w_{e_{2}}=\left[\begin{array}{c}
0.0033 \\
0.0138 \\
0.0819 \\
0.0369 \\
1
\end{array}\right]
$$

and

$$
b_{e_{2}}=\left[\begin{array}{c}
0 \\
0.1048 \\
0.2321 \\
0.2079 \\
0.1711
\end{array}\right], \quad c_{e_{2}}=c_{o}
$$

Also this time "tilting" one of the coordinates is enough to achieve positivity of the left dominant eigenvector. However by acting on the fifth state component (instead of the fourth of Example 1) we obtain an eventually positive realization whose power index is 5 ( $A_{e_{2}}^{k} \geq 0$ already at $k=2$, then we must take further powers to get all positive entries).

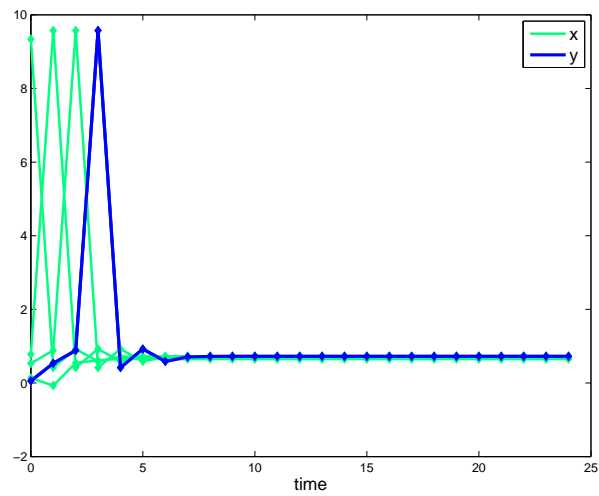

Fig. 2. Example 2. For the same $H(s)$ of Example 1, changing the transformation matrix $T$ may lead to a different eventually positive realization.

\section{RECOVERING POSITIVITY THROUGH DOWNSAMPLING}

The outcome of Algorithm 1, when successful, is a minimal eventually positive realization $\left\{A_{e}, b_{e}, c_{e}\right\}$, in which $A_{e} \stackrel{\vee}{>} 0$ and $b_{e} \geq 0, c_{e} \geq 0$. 
Remark 1: Notice that $b_{e} \geq 0 \Rightarrow \mathcal{R}_{e} \subset \mathbb{R}_{+}^{n}$, and hence $\Rightarrow x(k) \geq 0$ for $k \geq \eta_{o}$. It follows however from Proposition 2 that the impulse response of a minimum eventually positive realization with $b_{e} \geq 0$ and $c_{e} \geq 0$ is such that $x(t) \geq 0 \forall k \geq \eta_{o}, \forall x(0) \in \mathbb{R}_{+}^{n}$. In other words, while the impulse response completely characterize external positivity it is not enough to characterize internal positivity, as expected.

The realization $\left\{A_{e}, b_{e}, c_{e}\right\}$ can be used to obtain minimal positive realizations for decimated subsequences of the Markov parameters.

Theorem 6: Consider a strictly proper rational transfer function $H(z)$ satisfying Assumption 1 and the corresponding sequence of Markov parameters $\{h(k)\}_{k=1}^{\infty}$. Assume $\exists$ $r \in \mathbb{N}, r \neq 0$, such that $r \operatorname{Im}\left[\lambda_{i}-\lambda_{j}\right] \neq 2 \pi \xi, \xi=$ $\pm 1, \pm 2, \ldots$, for all distinct poles $\lambda_{i}$ and $\lambda_{j}$ of $H(z)$ having $\operatorname{Re}\left[\lambda_{i}-\lambda_{j}\right]=0$. If $H(z)$ admits a minimal eventually positive realization $\left\{A_{e}, b_{e}, c_{e}\right\}$ such that $b_{e} \geq 0$ and $c_{e} \geq 0$, then $\exists s \in \mathbb{N}$ such that the decimated sequence of Markov parameters $\left\{h_{s}(k)=h((k-1) s+1)\right\}_{k=1}^{\infty}$ admits a minimal positive realization.

Proof: Consider a minimal eventually positive realization $\left\{A_{e}, b_{e}, c_{e}\right\}$ of $H(z)$. If $\eta_{o}$ is the power index of $A_{e}$, $A_{e}^{s}>0 \forall s \in \mathbb{N}, s \geq \eta_{o}$. Then each decimated sequence $\left\{h_{s}(k)\right\}_{k=1}^{\infty}$ admits a positive realization $\left\{A_{s}, b_{e}, c_{e}\right\}$, where $A_{s}=A_{e}^{s}$. In fact, from $h(k)=c_{e} A_{e}^{k-1} b_{e}$, one gets $h_{s}(k)=$ $h((k-1) s+1)=c_{e} A_{e}^{(k-1) s} b_{e}=c_{e} A_{s}^{k-1} b_{e}$. In order for $\left\{A_{s}, b_{e}, c_{e}\right\}$ to be minimal, it must be $\left\{A_{s}, b_{e}\right\}$ controllable, and $\left\{A_{s}, c_{e}\right\}$ observable. Since the system is SISO and $\left\{A_{e}, b_{e}\right\}$ controllable, $\left\{A_{e}, c_{e}\right\}$ observable by construction, the geometric multiplicity of each distinct eigenvalue has to be 1 (i.e., there is a single Jordan block associated to each distinct eigenvalue). Denote $\lambda_{1}, \ldots, \lambda_{m}, m \leq n$, the distinct eigenvalues of $A_{e}$, of multiplicities $\mu_{1}, \ldots, \mu_{m}$. Since the eigenvalues of $A_{s}$ are $\lambda_{i}^{s}, i=1, \ldots, m$, whenever a merge happens, i.e., $\lambda_{i}^{s}=\lambda_{j}^{s}$ for $i, j \in\{1, \ldots, m\}$, $i \neq j$, then controllability and observability of $\left\{A_{s}, b_{e}, c_{e}\right\}$ are lost, i.e., $\left\{A_{s}, b_{e}, c_{e}\right\}$ is a nonminimal realization of $\left\{h_{s}(k)\right\}_{k=1}^{\infty}$ (the Markov subsequence "looses rank" because of the decimation). However, if $\exists$ nonzero $r \in \mathbb{N}$ such that $r \operatorname{Im}\left[\lambda_{i}-\lambda_{j}\right] \neq 2 \pi \xi \xi= \pm 1, \pm 2, \ldots$, for all distinct eigenvalues of $A_{e}$ such that $\operatorname{Re}\left[\lambda_{i}-\lambda_{j}\right]=0$, then $h(k)$ and $h_{s}(k)$ admit minimal realizations of the same dimension. In this case, an admissible decimation factor is $s=\min _{k \in \mathbb{N}}$ s.t. $r k \geq \eta_{o}$ and $r k \operatorname{Im}\left[\lambda_{i}-\lambda_{j}\right] \neq 2 \pi \xi, \xi= \pm 1, \pm 2, \ldots$.

Remark 2: Notice that if $\left\{A_{e}, b_{e}, c_{e}\right\}$ is a minimal eventually positive realization of $H(z)$, then a Zero-Order Hold (ZOH) downsampling of (8) by a factor $s \in \mathbb{N}$ yields

$$
\begin{aligned}
A_{\mathrm{ZOH}, s} & =A_{e}^{s} \\
b_{\mathrm{ZOH}, s} & =\sum_{j=0}^{s-1} A_{e}^{s-j-1} b_{e} \\
c_{\mathrm{ZOH}, s} & =c_{e} .
\end{aligned}
$$

The realization $\left\{A_{\mathrm{ZOH}, s}, b_{\mathrm{ZOH}, s}, c_{\mathrm{ZOH}, s}\right\}$ need not be posi- tive. For it however, the Markov parameters are

$h_{\mathrm{ZOH}, s}(k)=c_{\mathrm{ZOH}, s} A_{\mathrm{ZOH}, s}^{k-1} b_{\mathrm{ZOH}, s}=c_{e} A_{e}^{(k-1) s} \sum_{j=0}^{s-1} A_{e}^{s-j-1} b_{e}$

i.e., they are not a subsequence of the original $c_{e} A_{e}^{k-1} b_{e}$.

When $b_{\mathrm{ZOH}, s} \geq 0$ we have the following straightforward corollary of Theorem 6 .

Corollary 2: Consider a minimal eventually positive realization $\left\{A_{e}, b_{e}, c_{e}\right\}$ of $H(z)$ such that $b_{e} \geq 0$ and $c_{e} \geq 0$. Assume $\exists r \in \mathbb{N}, r \neq 0$, such that $r \operatorname{Im}\left[\lambda_{i}-\lambda_{j}\right] \neq 2 \pi \xi$, $\xi= \pm 1, \pm 2, \ldots$, for all distinct eigenvalues $\lambda_{i}$ and $\lambda_{j}$ of $A_{e}$ having $\operatorname{Re}\left[\lambda_{i}-\lambda_{j}\right]=0$. If $\mathcal{R}_{e} \subset \mathbb{R}_{+}^{n}$, then for $s \geq \eta_{o}$ the $\mathrm{ZOH}$ downsampled realization (9) is positive and minimal.

Example 3: Consider again Example 1 and the $\left\{A_{e}, b_{e}, c_{e}\right\}$ eventually positive realization. Since the power index is $8, A_{s}=A_{e}^{8}$ and $\left\{A_{s}, b_{e}, c_{e}\right\}$ is a minimal positive realization of the $\left\{h_{8}(k)=h((8 k-7)\}_{k=1}^{\infty}\right.$ subsequence of Markov parameters. By plotting the resulting dynamics in correspondence of the same nonzero initial condition plus impulse input of Fig. 1, we clearly see (Fig. 3) that the downsampling "neglects" the transient response and hence avoids one or more of its states to become temporarily negative. Analogous considerations hold for the realization $\left\{A_{e_{2}}, b_{e_{2}}, c_{e_{2}}\right\}$ (the decimation factor used in passing from Fig. 2 to Fig. 3 is $s=2$ ).

\section{CONCLUSION}

In an attempt to fill the gap between externally and internally positive linear systems, a novel class of realizations in introduced in this paper. These correspond to state update matrices that are eventually positive, hence that become positive after a certain power. The constructive procedure we provide in the paper to obtain such minimal eventually positive realizations is easy and quite general and, in our experience, always terminating with success when applied to externally positive systems having a single strictly dominating real eigenvalue of multiplicity 1 . When the multiplicity is higher or when dominance is not strict, then the situation is more intricate and it is not clear whether eventual positivity can still play a key role. When the method is applicable, the insight into the structure of the systems that one gets through eventually positive realizations is considerable, as seen for example in our downsampling theorems.

\section{REFERENCES}

[1] C. Altafini and G. Lini. Predictable dynamics of opinion forming for networks with antagonistic interactions. Automatic Control, IEEE Transactions on, 60(2):342-357, Feb 2015.

[2] B.D.O. Anderson, M. Deistler, L. Farina, and L. Benvenuti. Nonnegative realization of a linear system with nonnegative impulse response. Circuits and Systems I: Fundamental Theory and Applications, IEEE Transactions on, 43(2):134-142, Feb 1996.

[3] L. Benvenuti and L. Farina. A tutorial on the positive realization problem. Automatic Control, IEEE Transactions on, 49(5):651-664, May 2004.

[4] A. Berman and R.J. Plemmons. Nonnegative matrices in the mathematical sciences. Classics in applied mathematics. Society for Industrial and Applied Mathematics, 1994.

[5] L. Farina and S. Rinaldi. Positive Linear Systems: Theory and Applications. A Wiley-Interscience publication. Wiley, 2000. 

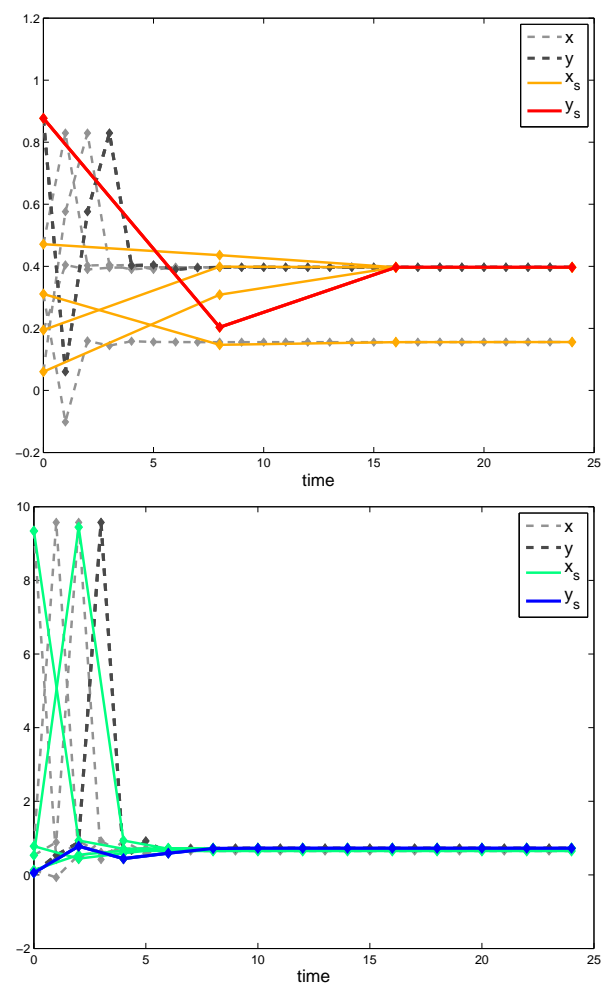

Fig. 3. Example 3. Dynamics of a downsampled minimal eventually positive realization. The response of the eventually positive system of Examples 1 (top) and 2 (bottom) and of the corresponding downsampled positive counterpart from the same initial condition are shown. The state and output of the downsampled positive realization $\left\{A_{s}, b_{e}, c_{e}\right\}$ are denoted resp. $x_{s}$ and $y_{s}$. The impulse response yields a positive output in both systems, and the downsampled system is also positive, but its transient contains less information than the original system.

[6] Shmuel Friedland. On an inverse problem for nonnegative and eventually nonnegative matrices. Israel Journal of Mathematics, 29(1):43-60, 1978.

[7] Charles R. Johnson and Pablo Tarazaga. On matrices with PerronFrobenius properties and some negative entries. Positivity, 8(4):327338, 2004.

[8] Dimitrios Noutsos. On Perron-Frobenius property of matrices having some negative entries. Linear Algebra and its Applications, 412:132 $-153,2006$.

[9] Yoshito Ohta, Hajime Maeda, and Shinzo Kodama. Reachability, observability, and realizability of continuous-time positive systems. SIAM Journal on Control and Optimization, 22(2):171-180, 1984.

[10] Dale D. Olesky, Michael J. Tsatsomeros, and Pauline van den Driessche. $M_{\vee}$-matrices: a generalization of M-matrices based on eventually nonnegative matrices. ELA. The Electronic Journal of Linear Algebra, 18:339-351, 2009.

[11] M. Valcher and L. Farina. An algebraic approach to the construction of polyhedral invariant cones. SIAM Journal on Matrix Analysis and Applications, 22(2):453-471, 2000. 